\title{
Percepción de los estudiantes de la Universidad Libre frente al fenómeno del embarazo en adolescentes de la ciudad de Cartagena
}

\author{
Rosario Cuadrado Álvarez ${ }^{1}$ \\ Leydis Patricia Santana Díaz \\ Massiel Miranda Dávila ${ }^{3}$
}

\begin{abstract}
Resumen
El embarazo en adolescentes es un fenómeno que aqueja a la sociedad de los países del tercer mundo, Colombia hace parte de dichos países por lo cual afronta esa problemática. Autoridades tales como la secretaria de interior y de cultura de Cartagena se han dado cuenta de la necesidad de afrontar dicho problema y la academia ha demostrado su preocupación a través de diversas investigaciones, algunas de las cuales se tratarán en el presente documento, algunas vistas desde la medicina otras desde lo social, pero todas intentan encontrar las causas del embarazo en los adolescentes y proponer soluciones.
\end{abstract}

\section{Palabras Claves}

Adolescencia, embarazo, fenómeno, madres solteras, acompañamiento familiar

\begin{abstract}
Pregnancy in adolescents is a phenomenon that afflicts the society of third world countries, Colombia is part of these countries and therefore faces this problem.Authorities such as the secretary of interior and culture of Cartagena have realized the need to address this problem and the academy has shown its concern through various investigations, some of which will be discussed in this document, some views from other medicine from the social, but all try to find the causes of pregnancy in adolescents and propose solutions.
\end{abstract}

\section{Keywords}

Adolescence, pregnancy, phenomenon, single mothers, family support

Fecha de recepción: 5 de Septirmbre de 2019, Fecha de evaluación: 17 de Septiembre de 2019, Fecha de aceptación: 14 Octubre de 2019

\footnotetext{
1. Administradora de Empresas, Especialista en Desarrollo Humano Organizacional, Magister en Desarrollo Humano Organizacional, $\mathrm{PhD}(\mathrm{c})$ en Ciencias Sociales Mención Gerencia. Docente de la Universidad Libre Cartagena Correo electronico: rosario.cuadrado@unilibrectg.edu.co.

2. Estudiante de 10 semestre de administración de la Universidad Libre Cartagena. Correo electronico: Leydis_santana@unili brectg.edu.co

3. Psicologa de la Escuela de Formación de Infanteria de Marina. Docente Investigadora.
} 


\section{Introducción}

La sociedad actual afronta diversos problemas que están afectando seriamente a los jóvenes, las drogas, el alcoholismo, los embarazos entre otros han llevado a nuestros jóvenes a ser catalogados como en situación de riesgo. Los entes gubernamentales vienen adelantado campañas que buscan educar a los jóvenes para evitar que los mismos sean arrastrados por dichos problemas. Se puede decir que el embarazo es uno de los temas más alarmantes, ya que las cifras indican que la sociedad se allá frente a un problema de salud pública, en la ciudad de Cartagena de Indias la cifras vienen en descenso desde 2007 con 4,684 pasos a 3,643 en 2016, aun las cifras son alarmantes y es de suma importancia el desarrollo de estrategias por parte del distrito más exactamente por la secretaria de interior, para reducir este problema de salud pública. La presente investigación busca analizar desde la perspectiva de los estudiantes de administración de empresas de la Universidad, cuáles son los factores con mayor incidencia para que se del embarazo en adolescentes.

\section{Aspectos conceptuales}

Para afrontar el eje de la investigación es necesario citar algunos conceptos que facilitaran la compresión y amplitud del estudio a desarrollar.

\section{Adolescencia}

García y Rodríguez (2014) afirman que

La adolescencia comienza en la biología y termina en la cultura, en algunas culturas comienza de los 12 o 13 años y termina finalizando los 19 o comenzando los 20. Por una parte, los procesos de la maduración dan lugar a una rápida aceleración del crecimiento físico, al cambio de las dimensiones del cuerpo, a modificaciones hormona- les y a unos impulsos sexuales más fuertes, al desarrollo de las características sexuales primarias y secundarias, así como a un nuevo crecimiento y diferenciación de las capacidades cognoscitivas. Estos desarrollos biológicos y la necesidad en que se ve el individuo de adaptarse a los mismos dan a la adolescencia algunas características universales y la separan de períodos anteriores al desarrollo.

Escobar y Muñoz (1995) citados por Merino y Rosales (2011) indican que

La adolescencia es un período de transición entre la niñez y la adultez. Su definición no es fácil, puesto que "su caracterización depende de una serie de variables: ubicación ecológica (referidos al medio ambiente natural), estrato socioeconómico, origen étnico y período de la adolescencia, entre otros."

Rodríguez (2013) asegura

La adolescencia es un período en el desarrollo biológico, psicológico, sexual, y social, inmediatamente posterior a la niñez y que comienza con la pubertad". Su rango de duración varía según las diferentes fuentes y opiniones médicas, científicas y psicológicas, pero generalmente se enmarca su inicio entre los 10 a 12 años, y su finalización a $\operatorname{los} 19$

Dice que la adolescencia es un período de transición o una etapa del ciclo de crecimiento que marca el final de la niñez y anuncia la adultez. Menacho (2005)

Blandón (2000), menciona que

La adolescencia abarca 3 etapas la cuales cumplen un periodo de tiempo desde los 10 a 13, lo cual denomina como adolescencia temprana, la adolescencia media que se encuentra comprendi- 


\section{Rosario Cuadrado Álvarez, Leydis Patricia Santana Díaz, Massiel Miranda Dávila}

da entre los años 14 a 16 años y finalmente la adolescencia tardía que abarca tres años comprendidos entre los 17 y 19 años.

Por su parte Echeverry y Grajales (2005) señalan que

Las etapas de la adolescencia se deben observar desde los factores biológicos, psicológicos e intelectuales. Además, indica que la adolescencia Temprana (10 a 13 años), Biológicamente, es el periodo peripuberal, con grandes cambios corporales y funcionales como la menarca. Psicológicamente el adolescente comienza a perder interés por los padres e inicia amistades básicamente con individuos del mismo sexo. Intelectualmente aumentan sus habilidades cognitivas y sus fantasías; no controla sus impulsos y se plantea metas vocacionales irreales.

Adolescencia media (14 a 16 años), es la adolescencia propiamente dicha; cuando ha completado prácticamente su crecimiento y desarrollo somático. Psicológicamente es el período de máxima relación con sus pares, compartiendo valores propios y conflictos con sus padres. Para muchos, es la edad promedio de inicio de experiencia y actividad sexual; se sienten invulnerables y asumen conductas omnipotentes casi siempre generadoras de riesgo. Muy preocupados por apariencia física, pretenden poseer un cuerpo más atractivo y se manifiestan fascinados con la moda.

Adolescencia tardía (17 a 19 años), casi no se presentan cambios físicos y aceptan su imagen corporal; se acercan nuevamente a sus padres y sus valores presentan una perspectiva más adulta; adquieren mayor importancia las relaciones íntimas y el gru- po de pares va perdiendo jerarquía; desarrollan su propio sistema de valores con metas vocacionales reales. Es importante conocer las características de estas etapas de la adolescencia, por las que todos pasan con sus variaciones individuales y culturales, para interpretar actitudes y comprender a los adolescentes especialmente durante un embarazo sabiendo que: "una adolescente que se embaraza se comportará como corresponde al momento de la vida que está transitando, sin madurar a etapas posteriores por el simple hecho de estar embarazada; son adolescentes embarazadas y no embarazadas muy jóvenes".

\section{Embarazo}

Rodríguez (2013) indica que

Es el período que transcurre entre la implantación en el útero del cigoto, el momento del parto en cuanto a los significativos cambios fisiológicos, metabólicos e incluso morfológicos que se producen en la mujer encaminados a proteger, nutrir y permitir el desarrollo del feto.

\section{Embarazo adolescente}

Echeverry y Grajales (2005) asegura que

Ocurre dentro de los dos años de edad ginecológica, entendiéndose por tal al tiempo transcurrido desde la menarca, y/o cuando la adolescente es aún dependiente de su núcleo familiar de origen". Los adolescentes tienen pensamientos y actitudes frente al embarazo, en los cuales se demuestra una baja percepción de la gravedad del evento, no observan daños potenciales para la salud, pero si muestran mayores preocupaciones de lo económico y la falta del apoyo parental. Algunos registran ganancias afec- 
tivas con el embarazo en la adolescencia, así como obtener compañía, mayor libertad, madurez y responsabilidad. Los adolescentes relatan del momento "ideal" para un embarazo, como el momento en que pueden tener estabilidad económica y no parece ser tan importante, sentirse preparado o desear el embarazo.

Barrozo y Pressiani (2011), aseguran que

El embarazo adolescente hace referencia a toda gestación que ocurre durante la adolescencia y comprende las mujeres de hasta 19 años, es decir, todo embarazo que ocurre dentro del tiempo transcurrido desde la menarca, y/o cuando la adolescente es aun dependiente de su núcleo familiar de origen. Las adolescentes que se embarazan, más que cualquier otra adolescente, necesitan apoyo, comprensión y ayuda, ya que está más sensible, insegura y desvalorizada que cualquier otra mujer y con mayor razón para una adolescente, que no se convierte en adulto por el hecho de ser madre. Para comprender como vive el embarazo una adolescente, se debe tener en cuenta que los cambios psicológicos, estarán condicionados por su historia de vida, por la relación con el progenitor de su hijo, por la situación con su familia, por su edad y especialmente por su madurez personal.

(Diaz., et al 2013), concluyen es su investigación

La prevalencia de adolecentes masculinos que han tenido experiencia de embarazos en sus parejas es muy similar a la de las mujeres. La asociación entre los factores predisponentes y el embarazo en adolescentes escolarizados no fue significativa estadísticamente. La edad en que más predomina el embarazo es a los 15 años; el nivelsocioeconómico donde se presentaron más embarazos fue en estratos 1y 2. La disfuncionalidad y funcionalidad familiar muestran datos parecidos con relación a la población que ha vivido una situación de embarazo. Los medios que les ofrecen mayor información a los adolescentes son las charlas educativas y la internet, siendo el embarazo y los métodos anticonceptivos temas de mayor interés. La mayoría de los sexualmente activos usan condón, sin embargo un porcentaje no se protege, siendo esto un riesgo para el embarazo no planificado y la propagación de enfermedades de transmisión sexual.

\section{Metodología}

\section{Tipo de investigación}

El tipo de investigación es descriptivo y exploratorio, se busca describir los aspectos que caracterizan el fenómeno de la adolescencia, reconociendo el mismo como un problema de salud pública. A demás de establecer un primer acercamiento al fenómeno de estudio.

\section{Enfoque de la investigación}

Enfoque de la investigación es mixto, se busca recopilar datos tanto cuantitativos como cualitativos, estos últimos nos permitirán observar diversas perspectivas.

\section{Población}

La población de estudio son los estudiantes de administración de empresas de la Universidad, cuyo número asciende a 384 estudiantes matriculados.

\section{Muestra}

El muestreo de la presente investigación se basa en los siguientes datos y se calcula con los siguientes formula. 
$\mathrm{N}=384$

$\mathrm{NC}=90 \%$

$\mathrm{Z}=1,64$

$\mathrm{P}=0,5$

$\mathrm{E}=0,05$

$n=\frac{N \cdot Z^{2} \cdot p(1-p)}{E^{2}(N-1)+Z^{2} \cdot p(1-p)}=\frac{(384) \cdot(1.64)^{2}(0.5)(1-0.5)}{(0.05)^{2}(384-1)+(1.64)^{2}(0.5)(1-0.5)}=117$

\section{Fuentes de datos}

\section{Secundarias}

Estudios de pregrado y maestría, que responden a los nombres

- Trabajo de Campo: Embarazo en Adolescentes Incidencia Prevención y Control

- Embarazo adolescente entre 12 y 19 años

- Características psicosociales de las madres adolescentes en tres instituciones de salud de Manizales durante febrero a julio de 2003

\section{Primarias}

Estudiantes adolescentes de administración la facultad de ciencia económicas administrabas y contables de la Universidad.

\section{Variables del estudio}

Tabla 1. Mapa de variables

\begin{tabular}{|l|}
\hline \multicolumn{1}{|c|}{ VARIABLE } \\
\hline Nivel de educación \\
\hline Núcleo familiar primario \\
\hline Ocupación \\
\hline Farmacodependencia \\
\hline Nivel socioeconómico \\
\hline
\end{tabular}

Fuente: Elaboración propia a partir de Echeverry y Grajales (2005)

Las variables empleadas en este estudio derivan de mapa de variables propuesto en la investiga- ción denominada "Características psicosociales de las madres adolescentes en tres instituciones de salud de Manizales durante febrero a julio de 2003.", realizada por Echeverry y Grajales (2005)

\section{Escala de medición}

Malave (2007) afirma que

La escala Likert es un tipo de instrumento de medición o de recolección de datos que se dispone en la investigación social para medir actitudes. Consiste en un conjunto de ítems bajo la forma de afirmaciones o juicios ante los cuales se solicita la reacción (favorable o desfavorable, positiva o negativa) de los individuos.

Es una escala fijada estructuralmente por dos extremos recorriendo un continuo desde favorable hasta desfavorable con un punto medio neutral para cada afirmación (Likert, 1932 citado por Casas, 1999)

\section{Alternativas o Puntos Tipo Likert}

Dentro de los aspectos constitutivos de la escala tipo likert, es importante resaltar las alternativas o puntos, que corresponden a las opciones de respuesta de acuerdo al instrumento seleccionado. A continuación, las alternativas y valores más usados:

\section{Alternativa A}

- Muy de Acuerdo.

- De Acuerdo.

- Ni de acuerdo ni en desacuerdo.

- En desacuerdo.

- Muy en desacuerdo.

\section{Alternativa B}

- Totalmente de Acuerdo.

- De Acuerdo. 
- Neutral.

- En desacuerdo.

- Totalmente en desacuerdo.

\section{Alternativa C}

- Definitivamente sí.

- Probablemente sí.

- Indeciso.

- Probablemente no.

- Definitivamente no.

\section{Alternativa D}

- Completamente verdadero.

- Verdadero.

- Ni falso ni verdadero.

- Falso.

- Completamente falso.

\section{Técnicas y procedimientos de recolección de}

\section{la información}

Se diseño una encuesta con la cual se evalúan las variables de orden cuantitativo y como complemento a lo anterior un último ítem permite recopilar las observaciones puntuales de la muestra objeto de estudio.

\section{Resultados}

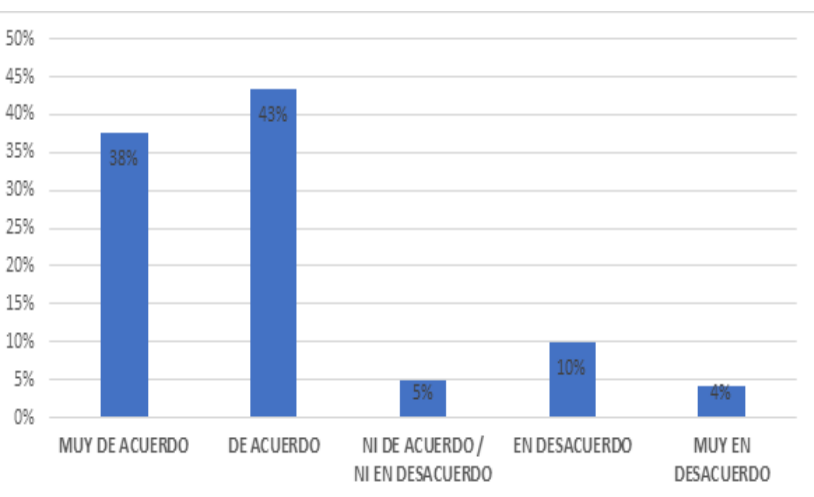

Gráfica 1. ¿Considera que el nivel de educación de los adolescentes es un aspecto determinante para evitar que el embarazo de adolescentes se presente?

Fuente: Elaboración propia

Los resultados a la anterior pregunta son $-38 \%$ muy de acuerdo, $43 \%$ de acuerdo, 5\% ni de acuerdo/ni en desacuerdo, $10 \%$ en desacuerdo, $4 \%$ muy en desacuerdo: Se observa que los estu- diantes consideran que el nivel educación es relevante para evitar los embarazos en adolescentes.

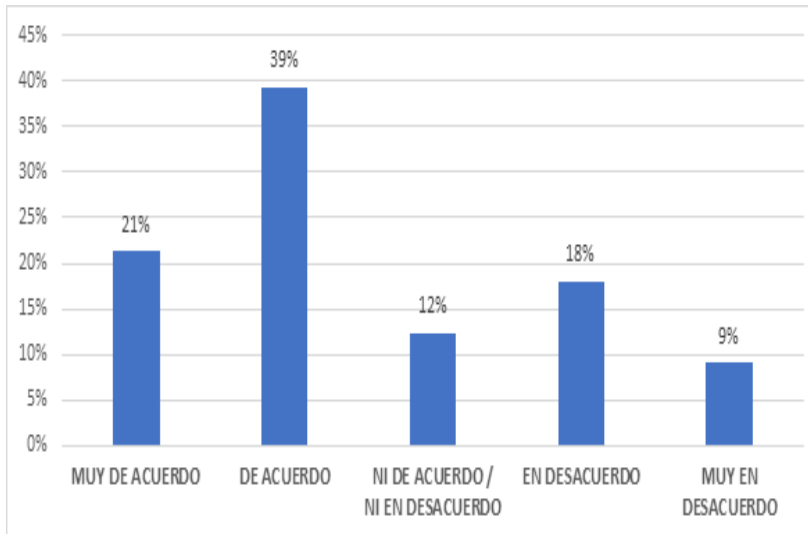

Gráfica 2. ¿Considera que el nivel de educación de los padres es un aspecto determinante para evitar que el embarazo de adolescentes se presente?

Fuente: Elaboración propia

Los resultados a la anterior pregunta son $-21 \%$ muy de acuerdo, $39 \%$ de acuerdo, $12 \%$ ni de acuerdo/ni en desacuerdo, $18 \%$ en desacuerdo, 9\% muy en desacuerdo: La educación de los padres es observada con un elemento fundamental para la guia sexual de los adolescentes.

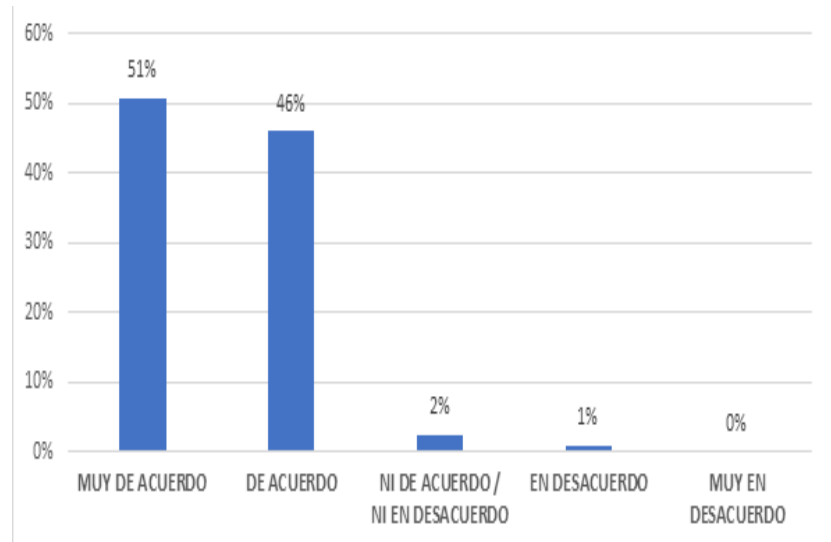

Gráfica 3. ¿Considera que es fundamental el apoyo del núcleo familiar para guiar en temas de sexualidad a sus hijos adolescentes?

Fuente: Elaboración propia

Los resultados a la anterior pregunta son $-51 \%$ muy de acuerdo, $46 \%$ de acuerdo, $2 \%$ ni de acuerdo/ni en desacuerdo, $1 \%$ en desacuerdo, $0 \%$ muy en desacuerdo: El apoyo del nucleo familiar es importante en el proceso de crecimiento de los adolescentes en materia de relaciones sexuales. 


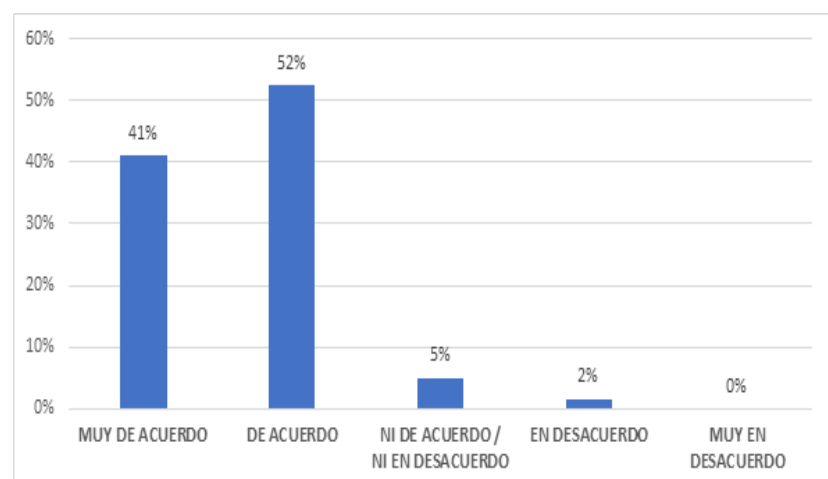

Gráfica 4. ¿Considera que el nivel socioeconómico esta asociado a los casos de embarazo en adolescentes?

Fuente: Elaboración propia

Los resultados a la anterior pregunta son $-41 \%$ muy de acuerdo, $52 \%$ de acuerdo, $5 \%$ ni de acuer- do/ni en desacuerdo, $2 \%$ en desacuerdo, $0 \%$ muy en desacuerdo: El nivel socioeconómico guarda gran relación con los casos de embarazos en adolescentes

\section{Conclusiones}

Es claro que aspectos como la educación, el nivel socioeconómico, el consumo de sustancia psicoactivas, son causantes de que el embarazo en adolescentes sea un mal que agobia a los jóvenes. Es necesaria el acompañamiento por parte de los padres de familia, antes de embarazo y mucho más después, la colaboración, afectiva, emocional, psicología y económica, para mejor la situación de los adolescentes.

\section{Referencia bibliográficas}

Barrozo y Pressiani (2011). Embarazo adolescente entre 12 y 19 años. Consultado el 3 de octubre de 2017. Disponible en: http://bdigital.uncu.edu.ar/objetos_digitales/5989/barrozo-mariela.pdf

Echeverry y Grajales (2005). Características psicosociales de las madres adolescentes en tres instituciones de salud de Manizales durante febrero a julio de 2003. Consultado el 4 de octubre de 2017. Disponible en: http://telesalud.ucaldas.edu.co/telesalud/Sitio\%20Web\%20ObGyn/Proyecto $\% 20$ Investigacion $\% 20$ madre $\% 20$ adolescente.htm

García y Rodríguez (2014). Trabajo de Campo: Embarazo en Adolescentes Incidencia Prevención y Control. Consultado el 4 de octubre de 2017. Disponible en: http://stadium.unad.edu.co/preview/UNAD.php?url=/bitstream/10596/2619/1/17413684.pdf

Briones, G. (1995). Métodos y Técnicas de Investigación para las Ciencias Sociales, $2^{\mathrm{a}}$. reimp., Ed. Trillas, México.

Issler (2001). Embarazo en la adolescencia. Revista de Postgrado de la Cátedra VIa Medicina 2001. N 107 - Agosto Página: 11-23

Merino y Rosales (2011). Factores que influyen en el embarazo de las adolescentes inscritas en el programa de atención materna de unidad de salud altavista, enero a junio de 2010. Consultado el 9 de octubre de 2017. Disponible en: http://ri.ues.edu.sv/11358/1/Factores\%20que\%20influyen $\% 20$ en $\% 20$ embarazo $\% 20$ adolescentes $\% 20$ enero $\% 202011$.doc

Rodríguez (2013). "Incidencia del embarazo en la adolescencia en mujeres de 12 a 18 años en maternidad mariana de Jesús” de septiembre 2012 a febrero del 2013. Consultado el 9 de octubre de 2017. Disponible en: http://repositorio.ug.edu.ec/bitstream/redug/1978/1/TESIS\%20VIVIANA\%20RODRIGUEZ\%20AQUINO.pdf 